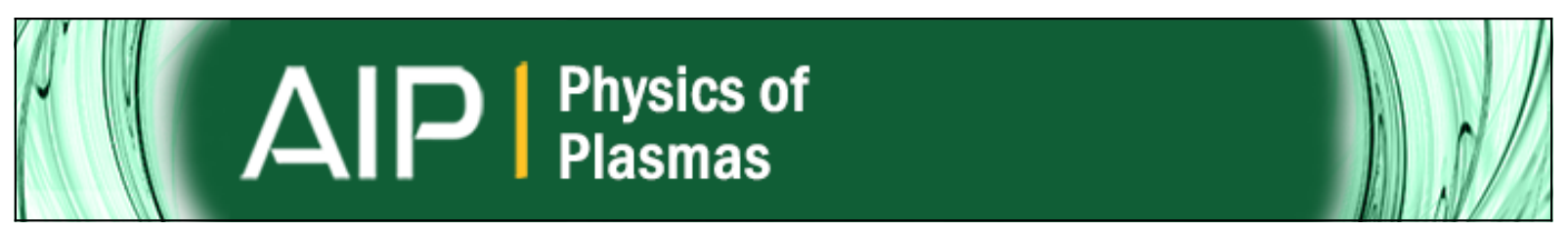

\title{
Cyclotron maser emission: Stars, planets, and laboratory a)
}

I. Vorgul, B. J. Kellett, R. A. Cairns, R. Bingham, K. Ronald, D. C. Speirs, S. L. McConville, K. M. Gillespie, and A. D. R. Phelps

Citation: Physics of Plasmas (1994-present) 18, 056501 (2011); doi: 10.1063/1.3567420

View online: http://dx.doi.org/10.1063/1.3567420

View Table of Contents: http://scitation.aip.org/content/aip/journal/pop/18/5?ver=pdfcov

Published by the AIP Publishing

\section{AIP Re-register for Table of Content Alerts}




\title{
Cyclotron maser emission: Stars, planets, and laboratory ${ }^{\text {a) }}$
}

\author{
I. Vorgul, ${ }^{1, b)}$ B. J. Kellett, ${ }^{2}$ R. A. Cairns, ${ }^{1}$ R. Bingham, ${ }^{2,3}$ K. Ronald, ${ }^{3}$ D. C. Speirs, ${ }^{3}$ \\ S. L. McConville, ${ }^{3}$ K. M. Gillespie, ${ }^{3}$ and A. D. R. Phelps ${ }^{3}$ \\ ${ }^{1}$ School of Mathematics and Statistics, University of St Andrews, KY16 9SS, United Kingdom \\ ${ }^{2} S T F C$ Rutherford Appleton Laboratory, Chilton, Didcot OX11 OQX, United Kingdom \\ ${ }^{3}$ SUPA, Department of Physics, University of Strathclyde, Glasgow G4 ONG, United Kingdom
}

(Received 19 November 2010; accepted 3 February 2011; published online 12 April 2011)

\begin{abstract}
This paper is a review of results by the group over the past decade on auroral kilometric radiation and similar cyclotron emissions from stars and planets. These emissions are often attributed to a horseshoe or crescent shaped momentum distribution of energetic electrons moving into the convergent magnetic field which exists around polar regions of dipole-type stars and planets. We have established a laboratory-based facility that has verified many of the details of our original theoretical description and agrees well with numerical simulations. The experiment has demonstrated that the horseshoe distribution does indeed produce cyclotron emission at a frequency just below the local cyclotron frequency, with polarization close to X-mode and propagating nearly perpendicularly to the beam motion. We discuss recent developments in the theory and simulation of the instability including addressing a radiation escape problem and the effect of competing instabilities, relating these to the laboratory, space, and astrophysical observations. C 2011 American Institute of Physics. [doi:10.1063/1.3567420]
\end{abstract}

\section{INTRODUCTION}

Cyclotron maser instabilities play an important role in devices designed to produce high power microwaves, such as gyrotrons, ${ }^{1}$ and are also thought to be involved in radio emissions from stars and planets. For the Earth the important phenomenon of this sort is auroral kilometric radiation ${ }^{2,3}$ (AKR) in which strong radio emissions occur in the auroral zone at a frequency close to the electron cyclotron frequency. ${ }^{4-6}$ Similar radio emission is observed from other magnetized planets, in particular Jupiter, ${ }^{7}$ and from magnetized stars. ${ }^{8}$ The essential physics involved is a resonant interaction between a wave and a particle, in our case an electron. The condition for such a resonance between a wave with frequency $\omega$ and parallel (to the ambient magnetic field) wavenumber $k_{\|}$and an electron with parallel velocity $v_{\|}$is

$$
\omega-k_{\|} v_{\|}=\frac{\omega_{c e}}{\gamma},
$$

where $\omega_{\mathrm{ce}}=\frac{e B}{m}$ is the electron cyclotron frequency and $\gamma$ is the usual relativistic factor. What it says is that the wave frequency, Doppler shifted as a result of its parallel motion, equals the cyclotron frequency, corrected for the effect of the relativistic mass shift. Since the electron is free to move along the field lines any parallel momentum gained or lost to the wave is simply transferred to the electron and so

$$
\frac{\Delta\left(m c^{2} \gamma\right)}{\Delta p_{\|}}=\frac{\omega}{k_{\|}}
$$

If we have weakly relativistic electrons so that $\gamma \approx 1$, then this implies that

\footnotetext{
a) Paper UI2 4, Bull. Am. Phys. Soc. 55, 331 (2010).

${ }^{\mathrm{b})}$ Invited speaker.
}

$$
\left(p_{\|}-\frac{m \omega}{k_{\|}}\right)^{2}+p_{\perp}^{2}=\text { const } .
$$

so that electrons move along a circle centered at $\frac{\omega}{k_{k}}$. For electromagnetic waves $\frac{\omega}{k_{\|}}$is generally much greater than typical electron velocities, so that when an electron interacts with a wave its motion is primarily perpendicular to the magnetic field. Whether a particular electron gains or loses energy when interacting with a wave depends on where it is with respect to the wave phase, so the result is diffusion of the electrons along the circular diffusion path. From this we see that what would be expected to produce cyclotron instability is a population inversion along the perpendicular direction. Note that small relativistic corrections do not change this conclusion, but they are important in Eq. (1). One type of distribution with a population inversion along the perpendicular direction is the loss cone distribution ${ }^{9-11}$ which for many years was a favored explanation for AKR. More recently, attention has focused on a distribution with a horseshoe or crescent shaped distribution in $\left(p_{\|}, p_{\perp}\right)$ space. ${ }^{12}$ This can be produced if a beam with an initial thermal spread moves along converging magnetic field lines, in which case conservation of energy and of magnetic moment gives rise to the horseshoe distribution. We should also mention another mechanism for instability which is important if there is an almost monoenergetic ring distribution of electrons, a situation more appropriate to a gyrotron than a naturally occurring plasma. Electrons whose phase means that they are accelerated by the wave gain energy and as a result their cyclotron frequency decreases because of the relativistic mass shift, while the opposite occurs for electrons which lose energy to the wave (Fig. 1). The result is a bunching of electrons in their gyro orbits, with more electrons feeding energy 

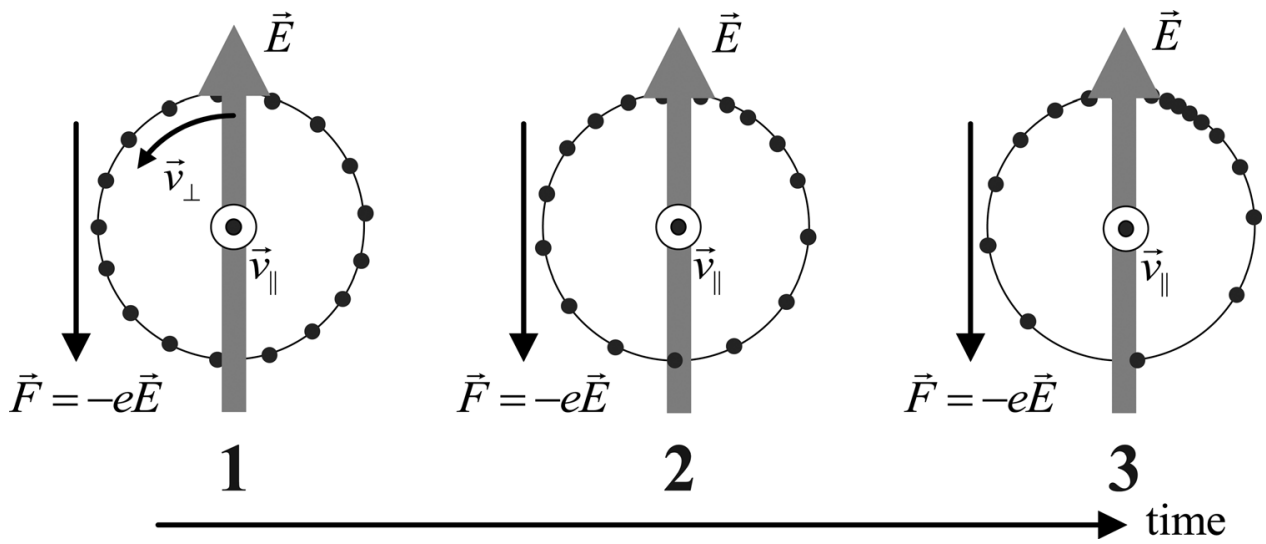

FIG. 1. Illustration of electron bunching in their gyro orbits.

into the electromagnetic wave than are absorbing energy from it. ${ }^{1}$ Our own program of research has involved a study of the horseshoe instability, combining theoretical analysis, ${ }^{13,14}$ application to the problem of emissions from planets and $\operatorname{stars}^{8}$ and an experimental program in which we attempt to scale these processes to a laboratory experiment. ${ }^{15}$ Here we shall review the main results of this program and discuss some of our recent work.

\section{STELLAR AND PLANETARY EMISSIONS}

Prompted by the first radio resolved image of a star (UV Ceti), ${ }^{16}$ we undertook a review of the $\mathrm{x}$-ray and radio observations of active late-type stars, in general, and UV Ceti, in particular. $^{8}$ One of the aspects of the observations that we particularly noted was the highly polarized [nearly 100\% right-hand circular (RCP)] radio outbursts (analogous to flares on the Sun) of UV Ceti. We also noted the high degree of correlation observed between the x-ray and radio luminosity of active late-type stars. ${ }^{17}$ Taken together, these observations strongly suggested that a new radio emission mechanism was responsible for most (and possibly all) of the radio emission of active late-type stars. The $\sim 100 \%$ RCP flares and the first image itself (showing that the radio emission was emerging from the poles of UV Ceti) led us to a new cyclotron maser emission mechanism driven by an electron beam entering the converging magnetic polar region of the star. The fact that this mechanism could easily generate the observed radio flux

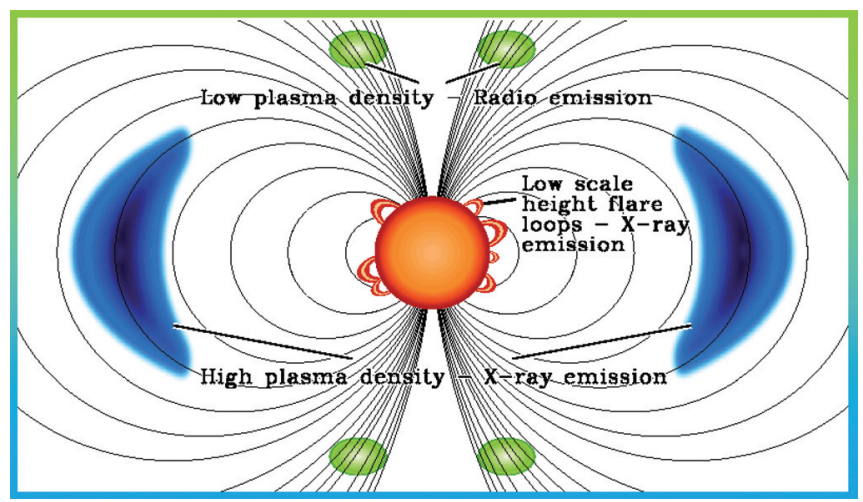

FIG. 2. (Color online) Diagrammatic representation of radiation emission from the flare star UV-Ceti (Ref. 8). from these stars with $\mathrm{keV}$ energy electrons could then also help explain the $\mathrm{x}$-ray/radio correlation. X-ray emission also implies keV electrons, so it was possible or even likely that the same electrons were responsible for the $\mathrm{x}$-ray and radio emission-therefore easily explaining the observed correlation. Our new model for UV Ceti ${ }^{8}$ and active late-type stars in general is summarized in Fig. 2.

More recently, we have made radio observations of $\mathrm{CU}$ Virginis, a quite different class of star-a magnetic chemically peculiar star. Figure 3 shows four successive nights of L band $(1.66 \mathrm{GHz}$ or $18 \mathrm{~cm}$ ) observation of CU Vir made by the Jodrell Bank MERLIN array. ${ }^{18}$ (The data are shown twice for clarity.) Detailed study of this star indicates that again it has a strong dipole field and that it rotates about an axis which is offset from the magnetic axis by an angle of around $70^{\circ 19}$ (Fig. 4).

All solar system planets which have strong magnetic fields also produce intense radio emission at frequencies close to the electron cyclotron frequency. The observations show the direct dependence of the radiation power on the intensity of the solar wind, ${ }^{20,21}$ with Jupiter being the most powerful radio source.

Stellar and planetary cyclotron maser emission is a significant source of information on the magnetospheres. It can

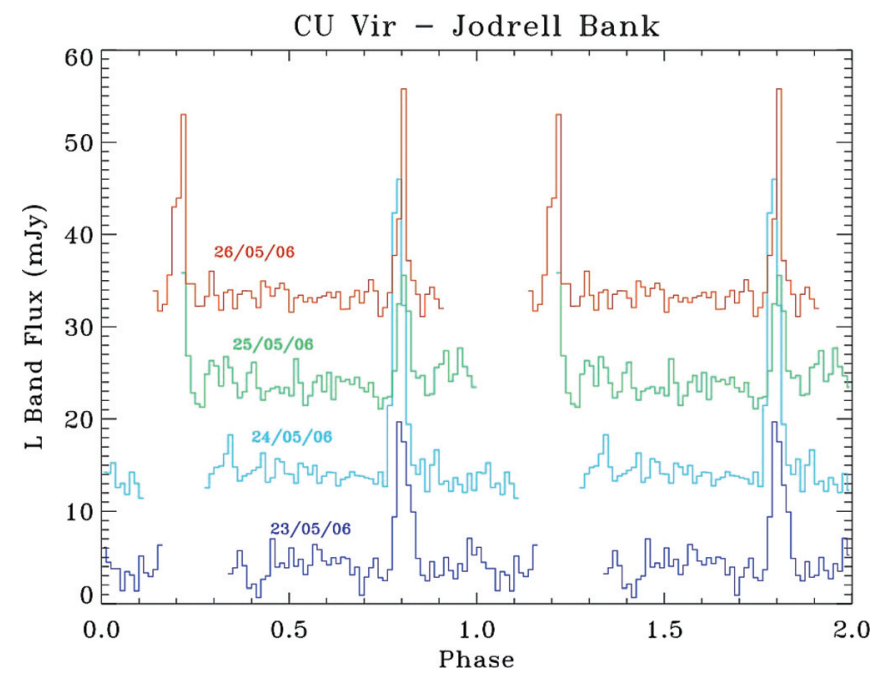

FIG. 3. (Color online) CU Virginis periodic $L$ band emission observed at Jodrell Bank telescope. 


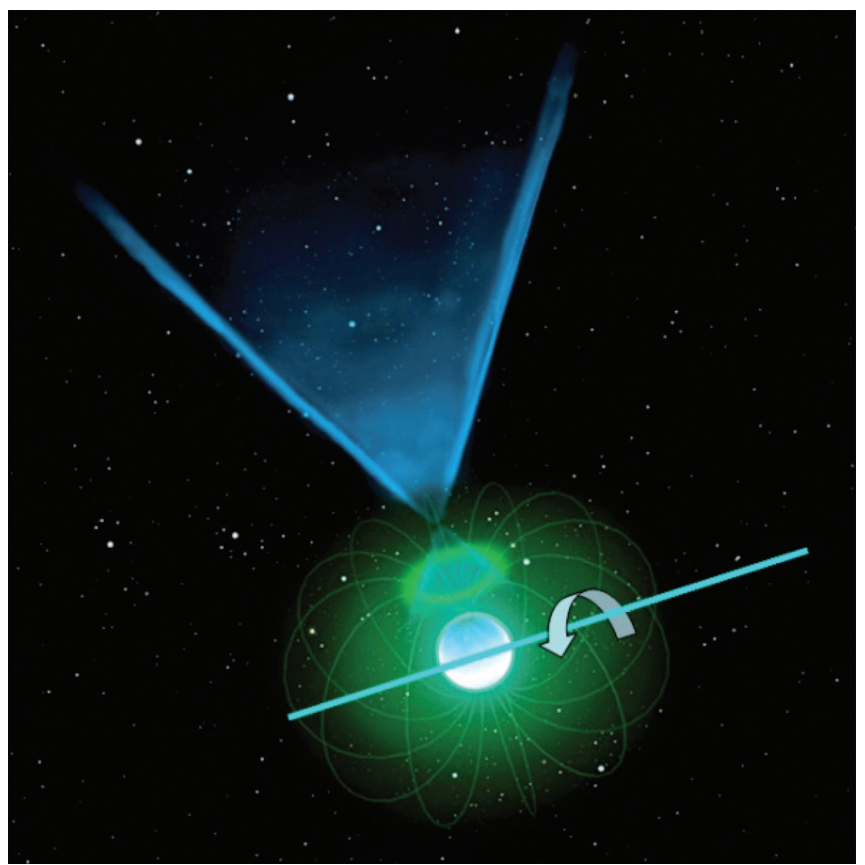

FIG. 4. (Color online) An artist impression of the star CU Virginis showing a geometry of rotation and a wide cone of radio emission emerging from one of the magnetic poles.

also allow us to study the angular momentum evolution of the stars and planets in order to detect further slowing down of them. In the case of CU Vir it helped to estimate the slowing down of its rotational period. ${ }^{22}$ Common features of such astrophysical radio sources are strong dipole magnetic field, existence of energetic electrons and observed radio emission propagating perpendicularly to the electron beams.

\section{SATELLITE OBSERVATIONS AND THE HORSESHOE SHAPED MOMENTUM DISTRIBUTION}

The easiest source for observation is Earth, and auroral kilometric radiation (AKR) has been observed in detail by different satellites. Satellite observations of the auroral emission from the magnetosphere indicate that it occurs in cavities of low background density aligned along the magnetic field. ${ }^{3,4}$

From the first images of the terrestrial auroral electron distribution function (DE-1 satelite ${ }^{23}$ ), a definite crescent, or what we call a horseshoe, can be seen. Later observations by the FAST satellite brought much clearer images where one can see an obvious highly populated horseshoe. ${ }^{2}$

The main results of the satellite observations relevant to cyclotron maser emission are the existence of fast electrons of approximately the same energy moving into a convergent magnetic field; an observed horseshoe shaped electron momentum distribution; intensive kilometric radiation nearly perpendicular to the electrons motion; sharp frequency spectrum around the electron cyclotron frequency; inside the aurora cavity the emission is polarized in the $\mathrm{X}$-mode; estimated efficiency of the emission is about $1 \%$.

It is now well established that AKR is a result of cyclotron emission. We suggested that the horseshoe shaped distribution function is responsible for this emission with the most important factor in forming such a distribution being a convergent magnetic field. With two invariants, energy, $v_{\perp}^{2}+v_{\|}^{2}=$ const . and magnetic moment (3), an initial drifting Maxwellian $f_{0}\left(v_{\|}, v_{\perp}\right)=A e^{-m / 2 T\left(\left(v_{\|}-v_{0}\right)^{2}+v_{\perp}^{2}\right)}$ is transformed into the distribution

$$
f\left(v_{\|}, v_{\perp}\right)=A e^{-m / 2 T\left(\left(\sqrt{v_{\|}^{2}+\left(1-B_{0} / B\right) v_{\perp}^{2}}-v_{0}\right)^{2}+\left(B_{0} / B\right) v_{\perp}^{2}\right)}
$$

with $\frac{\partial f}{\partial v_{\perp}}>0$.

Electrons here lose their parallel energy and increase their perpendicular energy, producing the characteristic horseshoe (Fig. 5). Figure 6 shows the results of numerical simulations with the PIC code KARAT, ${ }^{24}$ where the horseshoe formation evident as the beam moves into the stronger magnetic field, and its subsequent saturation after the emissions occur can be seen. For near perpendicular propagation the resonant electrons, determined by the cyclotron resonance condition (1), have almost constant energy and if the parameters are such that they lie along the inside of the horseshoe the system is unstable to cyclotron instability. They will move toward the central line of the beam producing the radiation and flattening the distribution.

\section{LABORATORY EXPERIMENT}

To reproduce AKR and similar emissions an experiment was constructed at the University of Strathclyde aimed at modeling the main features of the auroral cyclotron emission process, although scaled to centimeter rather than kilometer wavelength. It models the auroral cavity with a convergent magnetic field and an axially propagating electron beam and investigates the resulting instabilities and radiation.

Technically, it looks as shown in Fig. 7, where a detailed view of some aspects of the apparatus is presented. The essential feature of the experiment is the careful design of a series of coils in order to produce a smooth convergence of the magnetic field lines in the central cavity. ${ }^{25}$ This allows the electron beam emitted from an electron gun to follow an almost adiabatic trajectory along the field and form a horseshoe

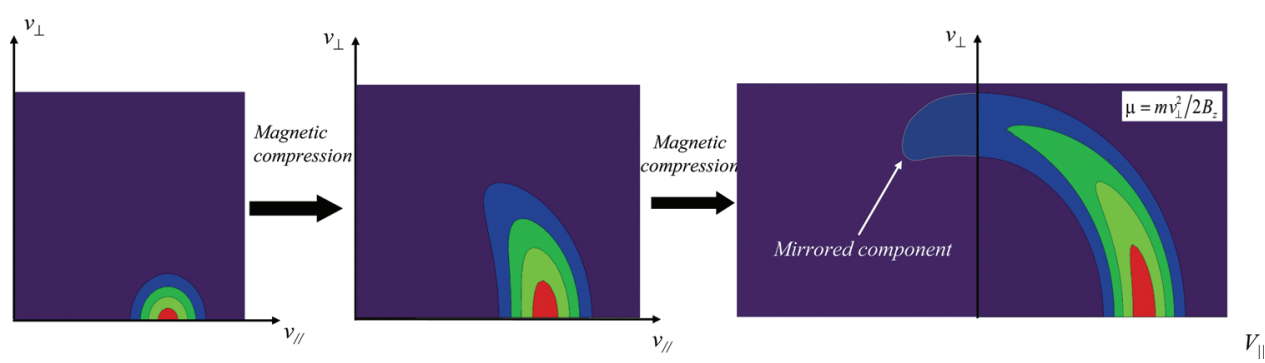

FIG. 5. (Color online) Illustration of the horseshoe distribution formation as electron beam with an initial energy spread moves into a stronger magnetic field. 


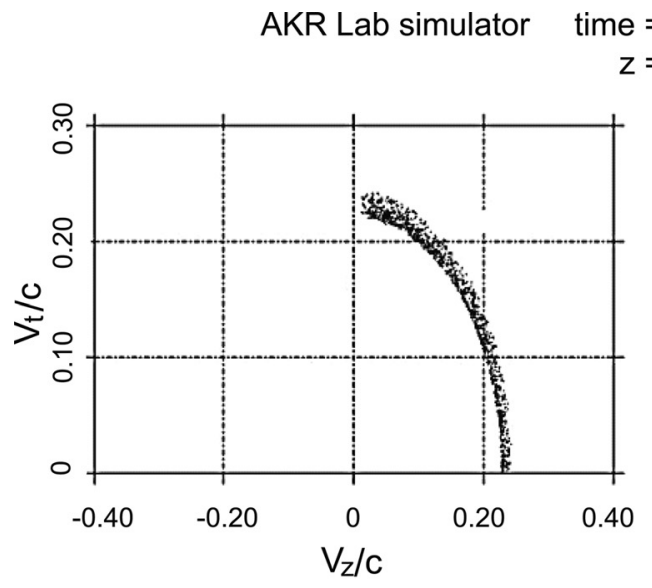

$$
\begin{aligned}
\mathrm{me} & =200.00 \mathrm{~ns} \\
\mathrm{z} & =6.0 \mathrm{~cm}
\end{aligned}
$$

AKR Lab simulator time $=200.00 \mathrm{~ns}$

$$
z=200.0 \mathrm{~cm}
$$
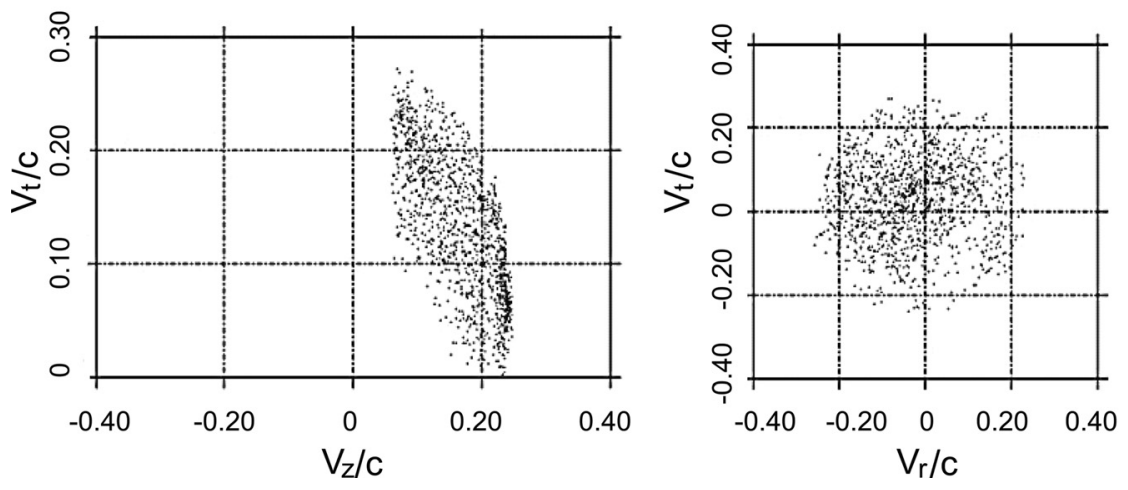

FIG. 6. KARAT simulation of horseshoe formation and evaluation with electron beam coming into stronger magnetic field (Ref. 24). distribution. The initial results from this experiment have been presented in the previous papers, ${ }^{15,26,27}$ so here we shall simply give a summary of the most important results. The main result is that narrow band emission around the cyclotron frequency is indeed produced as shown in Fig. 8(a) and that it is polarized in the X-mode in the same way as AKR. The generation of this radiation is as predicted by theory and is also in good agreement with computer simulations, an example of which is shown in Fig. 8(b). The results are in good agreement with the observations showing spectrally coherent radiation coming from a magnetically confined electron beam, with the radiation observed at near the electron cyclotron frequency.
Antenna measurements (Fig. 9) found that the radiated field was in the TE mode as predicted by numerical simulations and theory, with the measured modes having $T E_{03}$ and $T E_{23}$ harmonic structures. The efficiency with which electron beam energy is converted into electromagnetic wave energy was found to be in the range $1 \%-2 \%$ and in very good agreement with computer simulations. These simulations ${ }^{24,28,29}$ indicate that diffusion of the electrons toward lower perpendicular velocities is indeed an important property of the instability. A typical distribution as seen in Fig. 6 shows a clear quasilinear diffusion of the initially sharp horseshoe distribution evolving into a much more diffuse distribution with the horseshoe filled

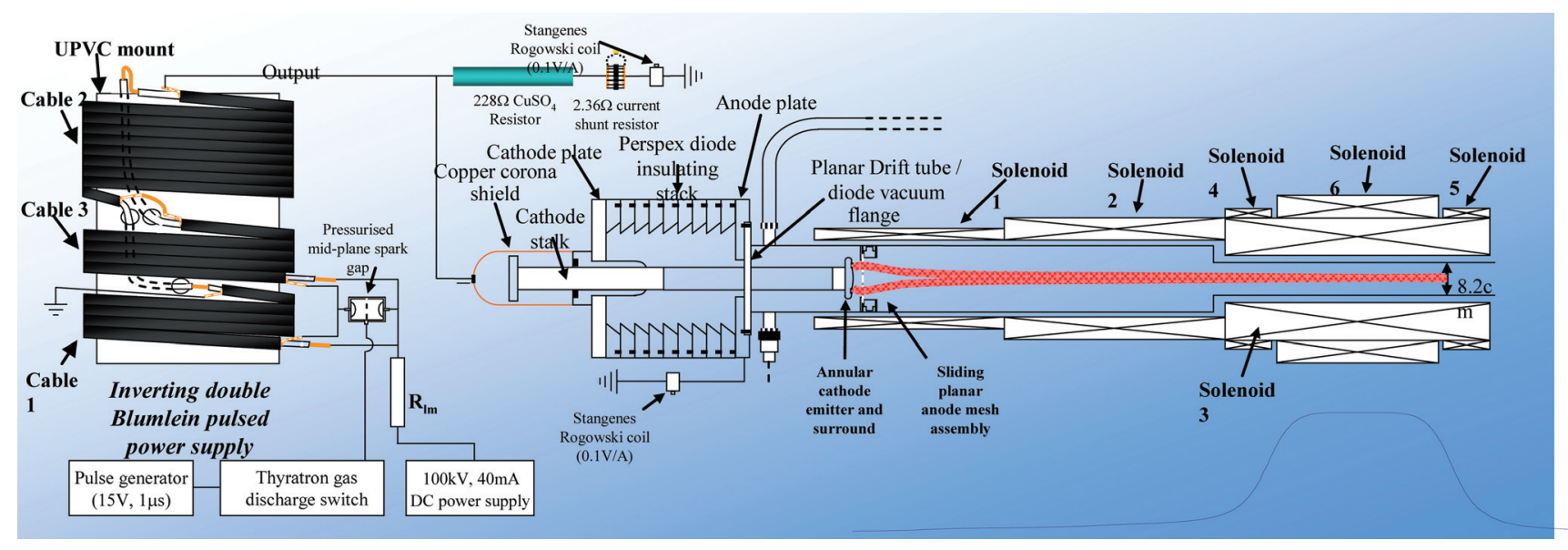

FIG. 7. (Color online) A scheme of experimental set up with magnetic field configuration along the electrons run plotted at the bottom of the solenoids arrangement. 


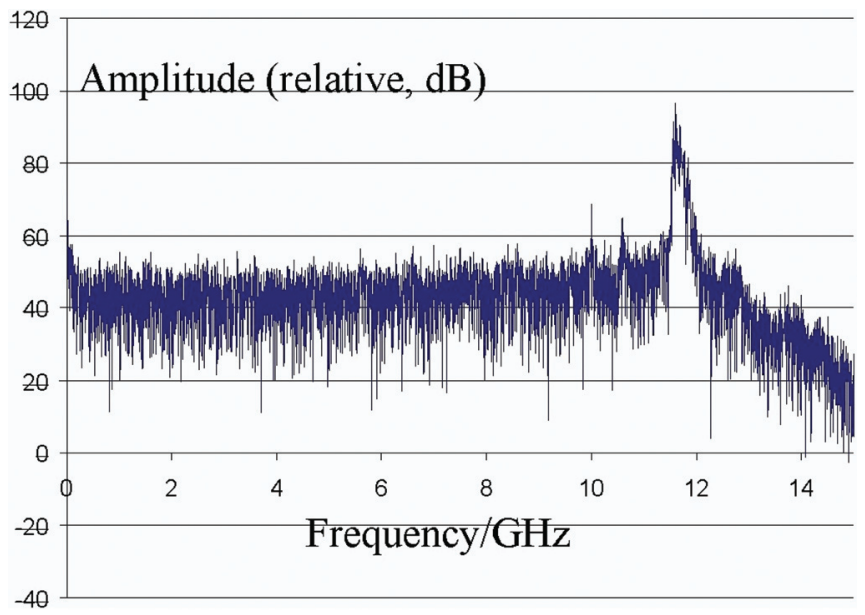

(a)

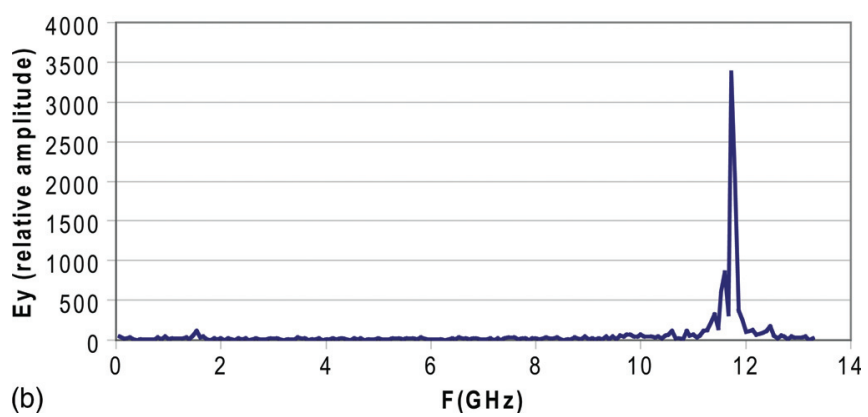

FIG. 8. (Color online) Cyclotron emission spectrum (a) produced by the experiment and (b) calculated using PIC KARAT.

in. There is also evidence of azimuthal bunching, so the instability appears to arise from a combination of the two mechanisms mentioned above. Estimates of the fraction of electron beam energy in an auroral cavity going into AKR also come up with a similar figure ${ }^{3}$ to the simulations and experiment. The broad agreement between the experimental results and the AKR observations suggest that the horseshoe-maser is indeed the dominant mechanism behind AKR.

The initial experiments used an electron beam sent into a vacuum, so the instability is purely the result of the horseshoe distribution of the beam rather than any interaction with a background plasma. However, more recently the apparatus has been modified to include such a plasma by introducing a Penning trap into the vacuum tube. Although observations show that auroral cavities are mostly populated by electrons, and the

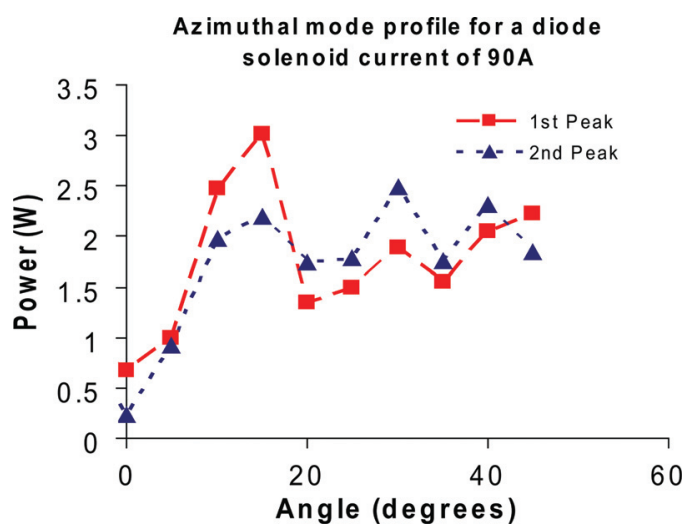

FIG. 9. (Color online) Antenna measurements for the maser cyclotron radiation. experiment as well as theory and simulations confirmed that the electron beams have the required free energy to explain the efficiencies observed in the polar magnetosphere, residual background plasma may still affect the radiation.

Results from exploring these effects are at a preliminary stage, but indications are that the growth of the instability is inhibited. However, the statistical variation in the emission appears to be somewhat unpredictable with very similar parameters giving quite different growth rates.

The reason for this sensitivity has not been fully explored but some preliminary calculations suggest that the two stream instability has a very similar growth rate to that of the horseshoe instability. It is perhaps the case that competition between these instabilities makes the evolution of the system very sensitive to the initial conditions. Further experimental and theoretical studies will be needed to decide if this is the correct explanation.

The introduction of a background plasma greatly extends the scope of the experiment and in future we intend to carry out investigation of a range of beam plasma instabilities of relevance to space and laboratory plasmas in a well controlled and well diagnosed experimental set up.

\section{THEORY}

We did theoretical modeling of the horseshoe radiation, relevant to the experiment and AKR. Detailed studies of the instability produced by an annular beam with a horseshoe velocity distribution have been carried out. The beam was taken to travel inside a conducting cylinder, a configuration intended to reproduce that of the experiment. We looked at modes in cylindrical geometry, with exact treatment of dispersion relations for the horseshoe distribution, getting the dispersion relations like the following: ${ }^{14}$

for TE modes

$$
\left(k_{11}-k_{z}^{2}\right)\left[k_{11}-k_{z}^{2}-\frac{\beta_{T E}^{2}}{R_{1}^{2}}\right]+k_{12}^{2}=0,
$$

and for TM modes

$$
\begin{array}{r}
{\left[k_{11}\left(1-\frac{k_{33} R_{1}^{2}}{\beta_{T M}^{2}}\right)+\frac{k_{z}^{2} k_{33} R_{1}^{2}}{\beta_{T M}^{2}}\right]\left(k_{11}-k_{z}^{2}-\frac{\beta_{T M}^{2}}{R_{1}^{2}}\right)} \\
+k_{12}^{2}\left(1-\frac{k_{33} R_{1}^{2}}{\beta_{T M}^{2}}\right)=0,
\end{array}
$$

where $k_{i j}$ are elements of the following dielectric tensor:

$$
\begin{aligned}
\hat{K}_{\perp} & =\left[\begin{array}{ll}
k_{11} & k_{12} \\
k_{21} & k_{22}
\end{array}\right] \\
& =\left(1-\frac{P_{e}^{2}}{\omega^{2}}\right) \hat{I}-\frac{P_{e}^{2}}{\omega^{2}} \int\left\{\frac{\hat{T}}{k_{z} v_{\|}-\omega+\Omega \sqrt{1-\left(v_{\|}^{2}+v_{\perp}^{2}\right) / c^{2}}}\right. \\
& \left.\cdot\left(-\frac{n \Omega}{v_{\perp}} \frac{\partial f}{\partial v_{\perp}}+k_{z} \frac{\partial f}{v_{\|}}\right) \frac{1}{n_{0}}\right\} d \vec{v},
\end{aligned}
$$


where for small Larmor radius, $\hat{T}=\frac{1}{4} v_{\|}^{2}\left[\begin{array}{ll}1 & -i \\ i & -1\end{array}\right]$ and $k_{i 3}=k_{3 j}=0, k_{33}=\left(1-\frac{P_{e}^{2}}{\omega^{2}}\right)$, with function $f$ being the horseshoe distribution function (3).

The results showed growth rates consistent with earlier estimates and with the computer simulations referred to above. A significant growth rate was found for several spatial modes [Fig. 10(a)]. Theoretical modeling found that the modes with higher growth rate have parallel component of the electric field much smaller than perpendicular one, that is, the most growing modes have quasi TE-mode structure, although, in general, the field inside the plasma region is found to be coupled TE and TM modes [Fig. 10(b)]. Growing modes propagate almost but not exactly perpendicular at about $4^{\circ}$ angle to the normal propagation which is in very good agreement with observations. A sharp frequency resonance was found at a frequency just below cyclotron frequency.

Resonant emission means that mostly the electrons which are close to the cyclotron resonance circle inside the horseshoe are producing the radiation. We did quasilinear estimation of the horseshoe evolution and found it being flat-
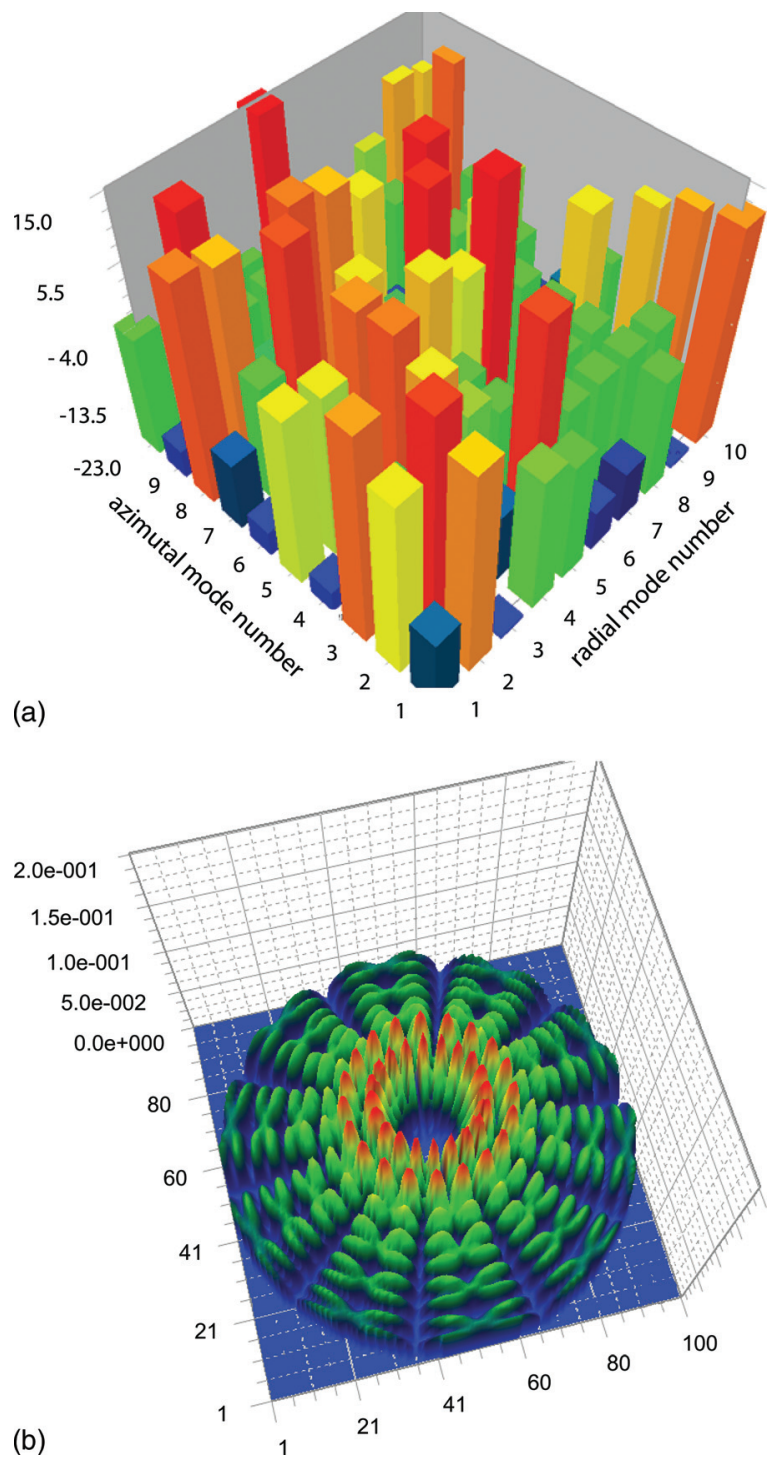

FIG. 10. (Color online) (a) Spatial modes growth rates and (b) typical spatial field structure for annular beam radiation. tening mostly around the value corresponding to the cyclotron frequency (see Fig. 11). We make estimation of the energy efficiency under quasilinear approximation from comparing the initial horseshoe distribution with the saturated one and found it being under $2 \%$ with small variations depending on the parameters.

An interesting feature is that there appear to be multiple mode structures within the annulus, all with comparable growth rates as shown in Fig. 10(a). These results came from numerical solution of a complicated dispersion relation and to understand the behavior in more detail we have recently looked at a simple slab geometry with a ring distribution, chosen because it is much easier to deal with. ${ }^{30}$

As can be seen from these results there are multiple modes with different wavelengths within the plasma slab. However, they all have very similar frequencies and hence couple to essentially the same wavelength outside the slab. They also have similar growth rates, so the behavior is very similar to that found previously for the horseshoe.

We addressed the problem of radiation escape in AKR theory which is that of how the radiation, generated at frequencies below the upper hybrid resonance, gets on to the higher frequency branch of the dispersion relation which connects to the regime of vacuum propagation. We looked at some of the dispersion properties of waves in the presence of energetic particles populations. For the equilibrium distribution function a monoenergetic ring, evaluating the standard dielectric tensor gives a dispersion equation which has real coefficients. For the case of growing modes, we should expect complex conjugate roots. Solving the dispersion equation showed that where the imaginary part disappears, the real part splits into two branches. This way the stable branches appear joining the two unstable ones. For the more realistic horseshoe distribution function rather than the monoenergetic ring results of numerical calculations look qualitatively similar to ring distribution. That suggests that a possible answer to the radiation escape problem could be the beam changes topology of dispersion curves, but this is a topic still under investigation.

As we have already mentioned, estimates of the growth rate for the two stream instability suggest that for the parameters of the experiment it is very similar to that of the horseshoe instability. In the future we intend to carry out a more

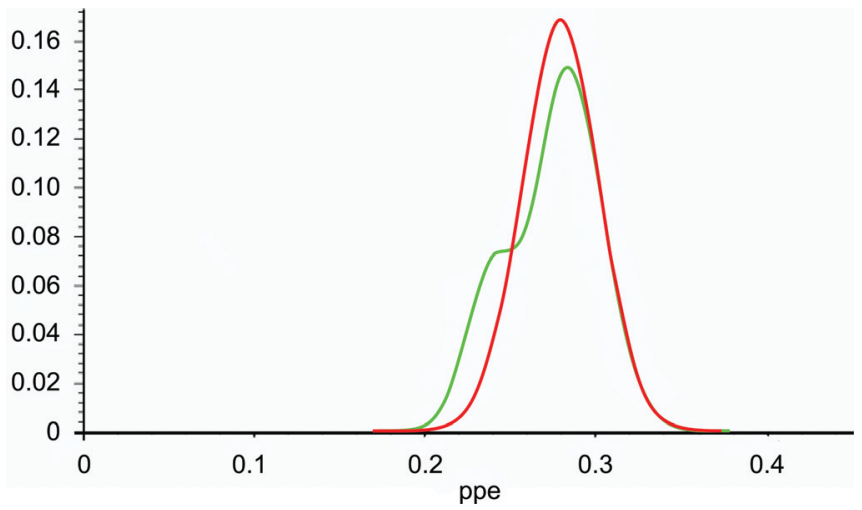

FIG. 11. (Color online) Quasilinear horseshoe saturation. 
detailed study of the stability of the horseshoe beam in the presence of a background plasma. We shall also study other possible instabilities which might be produced experimentally with a view to identifying appropriate parameter ranges within which phenomena of interest to the space plasma and fusion communities might be explored with our experiment.

\section{CONCLUSIONS}

Recognizing that a horseshoe velocity distribution will occur whenever an electron beam with a thermal spread travels along converging field lines, we have suggested that it may be an important factor in producing auroral kilometric radiation as well as radio emissions from other stars and planets. Theory and computer simulations show that the horseshoe distribution is unstable with a substantial growth rate. The theory only depends on the relative sizes of the wave frequency, the cyclotron frequency, and the plasma frequency, suggesting that a scaled laboratory experiment could be designed to reproduce the essential features of AKR with the emission in centimeter rather than kilometer wavelengths. Such an experiment has been designed and built and shown to produce radiation with the same spectral characteristics, direction, and polarization as AKR and with an efficiency of conversion of electron beam energy to radiation of the same order as that estimated for AKR. Building on the success of this program we have modified the apparatus to include a background plasma in addition to the beam. It resulted in producing a reduced power radiation with statistical variation in wave production which we suggest explaining by competition with two-stream instability. Addressing the radiation escape problem, we looked at possible ways of connecting to the vacuum propagation mode like the beam changing topology of dispersion curves. Future work on exploring other kinds of instabilities occurring at similar settings relevant to astrophysical as well as laboratory plasma is planned in experiment, theory, and computer simulations.

\section{ACKNOWLEDGMENTS}

The authors would like to thank the EPSRC and the STFC Centre for Fundamental Physics for their sponsorship of this research. The research was supported by UK Engineering and Physical Sciences Research Council.

${ }^{1}$ K. R. Chu, Phys. Fluids 21, 2354 (1978).

${ }^{2}$ G. T. Delory et al., Geophys. Res. Lett. 25, 2069, doi:10.1029/98GL00705 (1998).

${ }^{3}$ R. E. Ergun et al., Ap. J. 538, 456 (2000).

${ }^{4}$ R. J. Strangeway et al., Phys. Chem. Earth 26, 145 (2001).

${ }^{5}$ P. L. Pritchett and R. J. Strangeway, J. Geophys. Res. 90, 9650, doi:10.1029/JA090iA10p09650 (1985).

${ }^{6}$ P. L. Pritchett, R. J. Strangeway, R. E. Ergun, and C. W. Carlson, J. Geophys. Res. 107, 1437, doi:10.1029/2002JA009403 (2002).

${ }^{7}$ P. Zarka, J. Geophys. Res. 103, 20, doi:10.1029/98JE01323 (1998).

${ }^{8}$ B. J. Kellett et al., Mon. Not. R. Astron. Soc. 329, 102 (2002).

${ }^{9}$ G. S. Wu and L. C. Lee, Astrophys. J. 230, 621 (1979).

${ }^{10}$ L. C. Lee and G. S. Wu, Phys. Fluids 23, 1348 (1980).

${ }^{11}$ D. Le Quéau, R. Pellat, and A. Roux, Phys. Fluids 27, 247 (1984).

${ }^{12}$ R. Bingham and R. A. Cairns, Phys. Plasmas 7, 3089 (2000).

${ }^{13}$ R. Bingham, R. A. Cairns, and B. J. Kellett, Astron. Astrophys. 370, 1000 (2001).

${ }^{14}$ I. Vorgul, R. A. Cairns, and R. Bingham, Phys. Plasmas 12, 122903 (2005).

${ }^{15}$ D. C. Speirs et al., Phys. Plasmas 17, 056501 (2010).

${ }^{16}$ A. O. Benz et al., Astron. Astrophys. 331, 596 (1998).

${ }^{17}$ M. Güdel, J. H. M. M. Schmitt, and A. O. Benz, Astron. Astrophys. 302, 775 (1995).

${ }^{18}$ B. J. Kellet, V. Graffagnino, R. Bingham, T. W. B. Muxlow, and A. G. Gunn, e-print astro-ph/0701214 (2007).

${ }^{19}$ A. Hatzes, Mon. Not. R. Astron. Soc. 288, 153 (1997).

${ }^{20}$ M. D. Desch and M. L. Kaiser, Nature 310, 755 (1984).

${ }^{21}$ P. Zarka, Adv. Space Res. 12, 99 (1992).

${ }^{22}$ C. Trigilio et al., Mon. Not. R. Astron. Soc. 384, 1437 (2008).

${ }^{23}$ J. D. Menietti and J. L. Burch, J. Geophys. Res. 90, 5345, doi:10.1029/ JA090iA06p05345 (1985).

${ }^{24}$ D. C. Speirs, I. Vorgul, K. Ronald, R. Bingham, R. A. Cairns, A. D. R. Phelps, B. J. Kellett, A. W. Cross, C. G. Whyte, and C. Robertson, J. Plasma Phys. 71, 665 (2005).

${ }^{25}$ K. Ronald et al., Plasma Sources Sci. Technol. 17, 035011 (2008).

${ }^{26}$ K. Ronald et al., Phys. Plasmas 15, 056503 (2008).

${ }^{27}$ S. L. McConville et al., Plasma Phys. Controlled Fusion 50, 074010 (2008).

${ }^{28}$ D. C. Speirs et al., Plasma Phys. Controlled Fusion 50, 074011 (2008).

${ }^{29}$ K. M. Gillespie et al., Plasma Phys. Controlled Fusion 50, 124038 (2008).

${ }^{30}$ R. A. Cairns et al., Phys. Scr. T116, 23 (2005). 